\title{
Graphene disk in a solenoid magnetic potential: Aharonov-Bohm effect without a two-slit-like setup
}

\author{
Adam Rycerz $\odot$ and Dominik Suszalski \\ Institute for Theoretical Physics, Jagiellonian University, Łojasiewicza 11, PL-30348 Kraków, Poland
}

(Received 8 April 2020; revised manuscript received 29 May 2020; accepted 2 June 2020;

published 22 June 2020)

\begin{abstract}
The Aharonov-Bohm effect allows one to demonstrate the physical meaningfulness of magnetic vector potential bypassing the current in zero magnetic field regions. In the standard (a two-slit-like) setup, a conducting ring is pierced by magnetic flux and the quantum interference for an electron passing simultaneously through the two ring arms is observed. Here we show, by analyzing transport via evanescent waves, that the ballistic Corbino disk in graphene subjected to a solenoid magnetic potential may exhibit conductance oscillations of the Aharonov-Bohm kind although the current flows through a single conducting element only.
\end{abstract}

DOI: 10.1103/PhysRevB.101.245429

\section{INTRODUCTION}

Quantum transport through the Corbino disk in graphene has been addressed both theoretically [1-7] and experimentally [8-12] by numerous authors, as the egde-free geometry allows one to probe the mesoscopic aspects of graphene, such as transport via evanescent waves [13], even in nanometerscale devices. At zero magnetic field, conductance of ultraclean ballistic disks as a fuction of the carrier concentration [10] shows good agreement with the basic mode-matching analysis of Ref. [1]. At nonzero field, periodic (approximately sinusoidal) magnetoconductance oscillations were predicted $[2,3]$ but experimental confirmation of such a remarkable quantum-interference phenomenon is missing.

Theoretical analysis of Ref. [2] employs the rotational symmetry of the problem, resulting in the total angular momentum conservation $\left(J_{z}=\hbar j\right.$, with $j= \pm 1 / 2, \pm 3 / 2, \ldots$, the angular-momentum quantum number). In the case of an undoped disk of the inner radius $R_{1}$ and the outer radius $R_{2}$, the Landauer-Büttiker transmission probabilities [14,15] read

$$
T_{j}=\frac{1}{\cosh ^{2}\left[\ln \left(R_{2} / R_{1}\right)\left(j+\Phi_{d} / \Phi_{0}\right)\right]},
$$

where $\Phi_{d}=\pi\left(R_{2}^{2}-R_{1}^{2}\right) B$ is the flux piercing the disk with a uniform magnetic field $B$, and $\Phi_{0}=2(h / e) \ln \left(R_{2} / R_{1}\right)$ defines the conductance-oscillation period. Further analysis shows that the formula equivalent to Eq. (1) can also be derived if the carrier concentration (hereinafter quantified by the Fermi energy $E_{F}$, with $E_{F}=0$ corresponding to the charge-neutrality point) is adjusted to any Landau level, $E_{n}=\operatorname{sgn}(n) v_{F} \sqrt{2|n| e B}$, with $n=0, \pm 1, \pm 2, \ldots$, and $v_{F} \approx$ $10^{6} \mathrm{~m} / \mathrm{s}$ being the energy-independent Fermi velocity in graphene.

Published by the American Physical Society under the terms of the Creative Commons Attribution 4.0 International license. Further distribution of this work must maintain attribution to the author(s) and the published article's title, journal citation, and DOI.
Away from Landau levels the transmission is strongly suppressed [2,7]. For instance, in the vicinity of the chargeneutrality point $(n=0)$ magnetoconductance oscillations may be observed in the magnetic field range limited by

$$
\left|\Phi_{d}\right| \lesssim \frac{2 h}{e} \ln \left(\frac{1}{k_{F} R_{1}}\right)=\frac{h}{e} \ln \left(\frac{1}{\pi\left|n_{C}\right| R_{1}^{2}}\right),
$$

where we have further defined $k_{F}=\left|E_{F}\right| /\left(\hbar v_{F}\right)$ and the outermost right equality follows from the relation between the Fermi wave number and the carrier concentration $\left(n_{C}\right)$, namely, $k_{F}=\sqrt{\pi\left|n_{C}\right|}$, including the fourfold (spin and valley) degeneracy of each quasiparticle level. On the other hand, the current flow through the system leads to carrier density fluctuations of the order of $\delta n_{C} \sim 1 /\left(\pi R_{2}^{2}\right)$, even in the absence of the charge inhomogeneity usually appearing due to the electron-hole puddle formation at low densities [16]. Taking the above as the lower bound to $\left|n_{C}\right|$ in Eq. (2), one immediately obtains $\left|\Phi_{d}\right| \lesssim \Phi_{0}$, suggesting it may be difficult (or even impossible) to observe the magnetoconductance oscillations in the linear-response regime. A proposal to overcome this difficulty by going beyond the linear-response regime was put forward [17].

A separate issue concerns the role of electron-electron interactions, which is usually marginal when discussing ballistic systems in monolayer graphene [18,19], in agreement with fundamental considerations [20,21], but may lead to Wigner crystallization or the appearance of fractional quantum Hall phases, in case the bulk density of states is strongly modified due to the Landau quantization [10,22,23].

Generally speaking, uniform magnetic fields, although being most feasible to generate at micrometer scale, do not seem to provide a realistic opportunity to observe magnetoconductance oscillations in graphene-based Corbino disks. Therefore it is worth to consider other field arrangemets, in which phase effects may overrule orbital effects (such as Landau level formation). In this paper, we focus on the case of a disk whose inner area is pierced by a long solenoid (see Fig. 1), generating the flux $\Phi_{i}$. Earlier, it was shown by Katsnelson [3,24] that for zero doping $\left(E_{F}=0\right)$ the transmission probabilities are given 


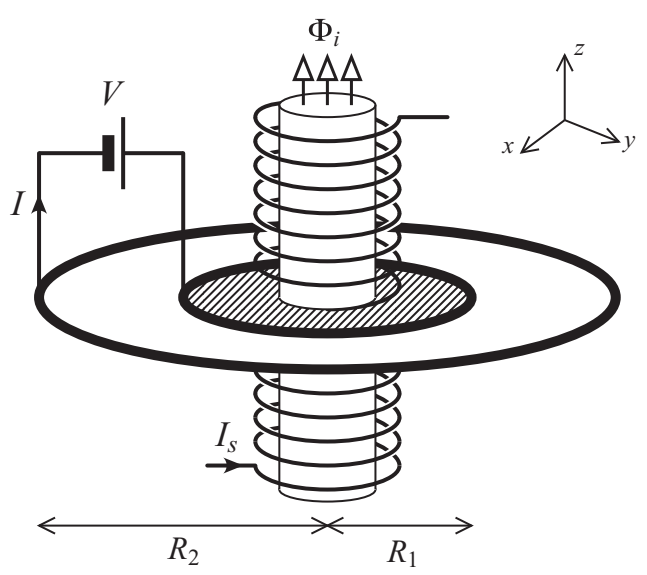

FIG. 1. Schematic of the Corbino disk in graphene of the inner radius $R_{1}$ and the outer radius $R_{2}$, contacted by two electrodes (thick black circles). A voltage source $(V)$ drives the current $(I)$ through the disk. A separate gate electrode (not shown) allows the carrier concentration in the disk to be tuned around the neutrality point. A long solenoid, carrying the current $I_{s}$, generates the flux $\Phi_{i}$ piercing the inner disk area. The coordinate system is also shown.

by Eq. (1) after substituting

$$
\Phi_{d} \equiv \Phi_{i} \quad \text { and } \quad \Phi_{0} \equiv \Phi_{\mathrm{AB}},
$$

with $\Phi_{\mathrm{AB}}=h / e$ being the familiar Aharonov-Bohm flux quantum [25]. However, the analysis of such a system away from the charge-neutrality point $\left(E_{F} \neq 0\right)$ is missing.

The remaining part of the paper is organized as follows. In Sec. II, we present the results of the mode-matching analysis for the system of Fig. 1 at arbitrary doping and flux. Next, in Sec. III, the numerical discussion of the conductance oscillations is provided. The effect of electrostatic field breaking cylindrical symmetry of the problem is considered in Sec. IV. The conclusions are given in Sec. V.

\section{SOLUTION FOR ARBITRARY DOPING AND FLUX}

Our analysis starts from the Dirac Hamiltonian in a single valley $(K)$, which is given by

$$
H=v_{F}(\boldsymbol{p}+e \boldsymbol{A}) \cdot \boldsymbol{\sigma}+U(r),
$$

where $\boldsymbol{p}=-i \hbar\left(\partial_{x}, \partial_{y}\right)$ is the in-plane momentum operator, the electron charge is $-e$, the magnetic vector potential of a solenoid is written in the symmetric gauge [26]

$$
\boldsymbol{A}=\left(A_{x}, A_{y}\right)=\frac{\Phi_{i}}{2 \pi}\left(-\frac{y}{r^{2}}, \frac{x}{r^{2}}\right),
$$

and $\sigma=\left(\sigma_{x}, \sigma_{y}\right)$ with $\sigma_{x}$ and $\sigma_{y}$ being the Pauli matrices. We further suppose that the electrostatic potential energy $U(r)$ depends only on $r=\sqrt{x^{2}+y^{2}}$; namely, we put $U(r)=0$ in the disk area $\left(R_{1}<r<R_{2}\right)$, or $U(r)=U_{\infty}$ otherwise. Since Hamiltonian (4) commutes with the total angular momentum operator, $J_{z}=-i \hbar \partial_{\varphi}+\hbar \sigma_{z} / 2$, the energy eigenfunctions can be chosen as eigenstates of $J_{z}$

$$
\psi_{j}(r, \varphi)=e^{i(j-1 / 2) \varphi}\left(\begin{array}{c}
\chi_{j, A}(r) \\
\chi_{j, B}(r) e^{i \varphi}
\end{array}\right),
$$

where $j$ is a half-odd integer, two spinor components $(A, B)$ correspond to the sublattice degree of freedom, and we have introduced the polar coordinates $(r, \varphi)$. The Dirac equation now can be written as $H_{j}(r) \chi_{j}(r)=E \chi_{j}(r)$, where $\chi_{j}(r)=$ $\left[\chi_{j, A}(r), \chi_{j, B}(r)\right]^{T}$, and

$$
\begin{aligned}
H_{j}(r)= & -i \hbar v_{F} \sigma_{x} \partial_{r}+U(r) \\
& +\hbar v_{F} \sigma_{y}\left(\begin{array}{cc}
\frac{j-1 / 2}{r}+\frac{e \Phi_{i}}{h r} & 0 \\
0 & \frac{j+1 / 2}{r}+\frac{e \Phi_{i}}{h r}
\end{array}\right) .
\end{aligned}
$$

For a piecewise-constant potential energy $U(r)$ and the electron-doping case, $E>U(r)$, the eigenfunctions of $H_{j}(r)$ (7) for the incoming (i.e., propagating from $r=0$ ) and outgoing (propagating from $r=\infty$ ) waves are given, up to the normalization, by

$$
\chi_{j}^{\text {in }}=\left(\begin{array}{l}
H_{v(j)-1 / 2}^{(2)}(k r) \\
i H_{v(j)+1 / 2}^{(2)}(k r)
\end{array}\right), \chi_{j}^{\text {out }}=\left(\begin{array}{c}
H_{v(j)-1 / 2}^{(1)}(k r) \\
i H_{v(j)+1 / 2}^{(1)}(k r)
\end{array}\right),
$$

where

$$
v(j)=j+\Phi_{i} / \Phi_{\mathrm{AB}},
$$

$H_{v}^{(1,2)}(\rho)$ is the Hankel function of the (first, second) kind, and $k=|E-U(r)| /\left(\hbar v_{F}\right)$. The solution for the disk area can be represented as

$$
\chi_{j}^{(d)}=A_{j} \chi_{j}^{\text {in }}\left(k_{F} r\right)+B_{j} \chi_{j}^{\text {out }}\left(k_{F} r\right), \quad R_{1}<r<R_{2},
$$

with $A_{j}$ and $B_{j}$ being arbitrary constants, and the Fermi wave number $k_{F}=|E| /\left(\hbar v_{F}\right)$. For the hole doping case, $E<U(r)$, the wave functions are replaced by $\tilde{\chi}_{j}^{\text {in(out) }}=\left[\chi_{j}^{\text {in(out) }}\right]^{\star}$, where we use the relation $H_{v}^{(2)}=\left[H_{v}^{(1)}\right]^{\star}$.

The heavily doped graphene leads are modeled here by taking the limit of $U(r)=U_{\infty} \rightarrow \pm \infty$ for $r<R_{1}$ or $r>R_{2}$. The corresponding wave functions can be simplified to

$$
\begin{gathered}
\chi_{j}^{(1)}=\frac{e^{ \pm i k_{\infty}}}{\sqrt{r}}\left(\begin{array}{l}
1 \\
1
\end{array}\right)+r_{j} \frac{e^{\mp i k_{\infty}}}{\sqrt{r}}\left(\begin{array}{c}
1 \\
-1
\end{array}\right), \quad r<R_{1}, \\
\chi_{j}^{(2)}=t_{j} \frac{e^{ \pm i k_{\infty}}}{\sqrt{r}}\left(\begin{array}{l}
1 \\
1
\end{array}\right), \quad r>R_{2},
\end{gathered}
$$

where we have introduced the reflection (transmission) amplitudes $r_{j}\left(t_{j}\right)$ and $k_{\infty}=\left|E-U_{\infty}\right| /\left(\hbar v_{F}\right) \rightarrow \infty$.

Solving the mode-matching conditions, $\chi_{j}^{(1)}\left(R_{1}\right)=$ $\chi_{j}^{(d)}\left(R_{1}\right)$ and $\chi_{j}^{(d)}\left(R_{2}\right)=\chi_{j}^{(2)}\left(R_{2}\right)$, we find the transmission probability for $j$ th mode

$$
T_{j}=\left|t_{j}\right|^{2}=\frac{16}{\pi^{2} k^{2} R_{1} R_{2}} \frac{1}{\left[\mathfrak{D}_{v(j)}^{(+)}\right]^{2}+\left[\mathfrak{D}_{v(j)}^{(-)}\right]^{2}},
$$

where $v(j)$ is given by Eq. (9) and

$$
\begin{aligned}
\mathfrak{D}_{v}^{( \pm)}= & \operatorname{Im}\left[H_{v-1 / 2}^{(1)}\left(k R_{1}\right) H_{v \mp 1 / 2}^{(2)}\left(k R_{2}\right)\right. \\
& \left. \pm H_{v+1 / 2}^{(1)}\left(k R_{1}\right) H_{v \pm 1 / 2}^{(2)}\left(k R_{2}\right)\right] .
\end{aligned}
$$

\section{RESULTS AND DISCUSSION}

The linear-response conductance is calculated according to the Landauer-Büttiker formula $[14,15]$

$$
G=\frac{I}{V}=g_{0} \sum_{j= \pm 1 / 2, \pm 3 / 2, \ldots} T_{j},
$$



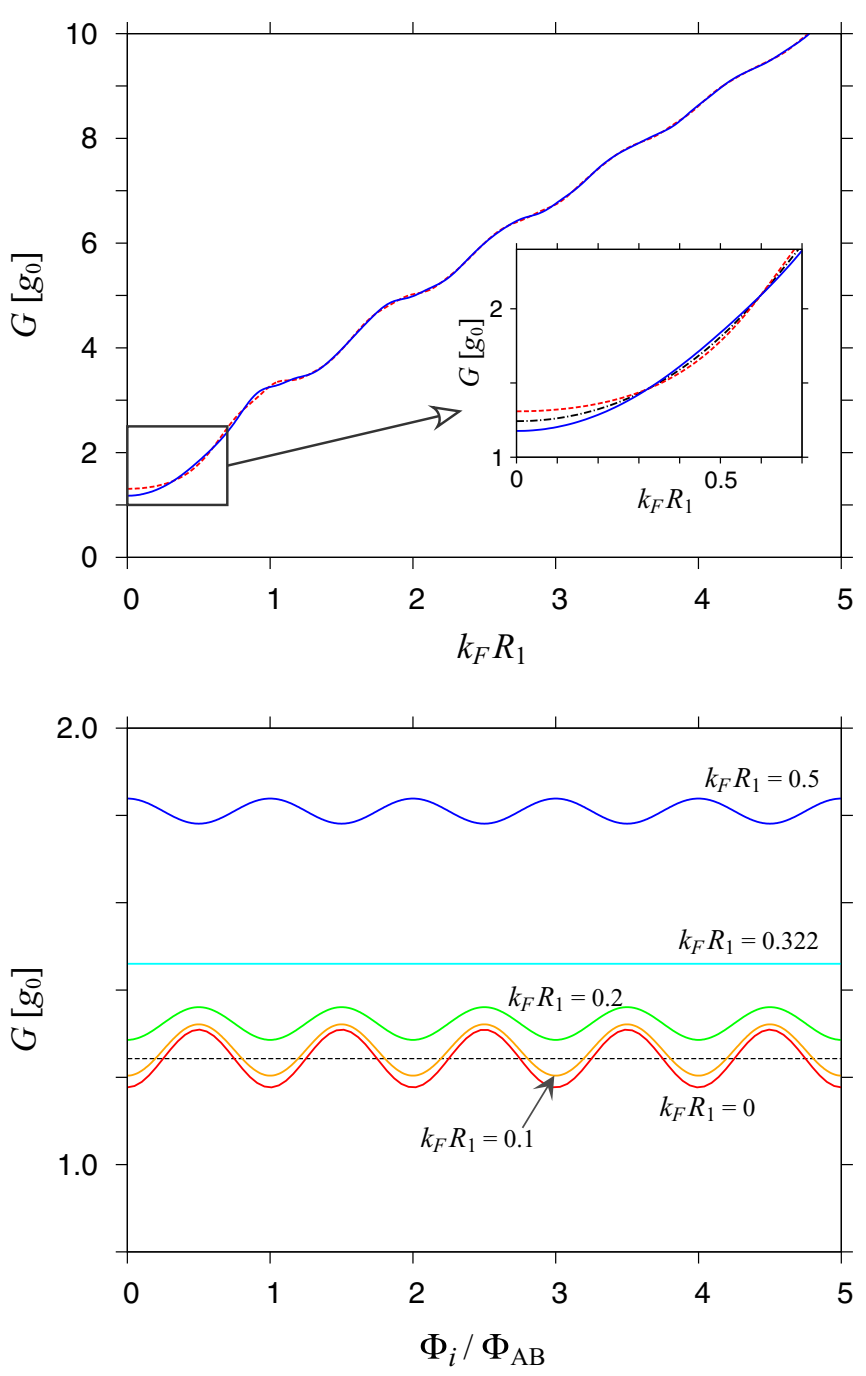

FIG. 2. Conductance as a function of the doping (top) and the flux piercing the inner disk area (bottom) for the radii ratio $R_{2} / R_{1}=$ 5. (Top) Different lines correspond to $\Phi_{i}=0$ (blue solid line) and $\Phi_{i}=\Phi_{A B} / 2$ (red dashed line). Inset presents a zoom-in, for low dopings, with an additional black dash-dot line depicting the conductance averaged over $\Phi_{i}$. (Bottom) The doping is varied from $k_{F} R_{1}=$ 0 to $k_{F} R_{1}=0.5$ and specified for each solid line on the plot. Dashed line marks the pseudodiffusive conductance $G_{\text {diff }}=2 g_{0} / \ln \left(R_{2} / R_{1}\right)$, with $g_{0}=4 e^{2} / h$.

where the conductance quantum $g_{0}=4 e^{2} / h$, with the factor 4 accounting for spin and valley degeneracy, and the summation over modes is performed numerically up to the machine round-off errors [27]. Our numerical results are presented in Figs. 2 and 3.

The asymptotic properties of the Hankel functions [28] in Eq. (13) lead to $T_{j} \approx 1$ for $k_{F} R_{1}-v(j) \gg 1$, with $v(j)$ given by Eq. (9), or to $T_{j} \approx 0$ for $v(j)-k_{F} R_{1} \gg 1$. In turn, the conductivity can by approximated as $G \approx 2 g_{0} k_{F} R_{1}$ for $k_{F} R_{1} \gg 1$ and $R_{2} \gg R_{1}$ (see top panel in Fig. 2), with an excess value of $\sim g_{0} R_{1} / R_{2}$ (up to the order of magnitude) representing the contribution from evanescent waves.

Furthermore, the structure of Eqs. (9), (13), (14), and (15) results in perfectly periodic functional dependence of $G(\Phi)$,
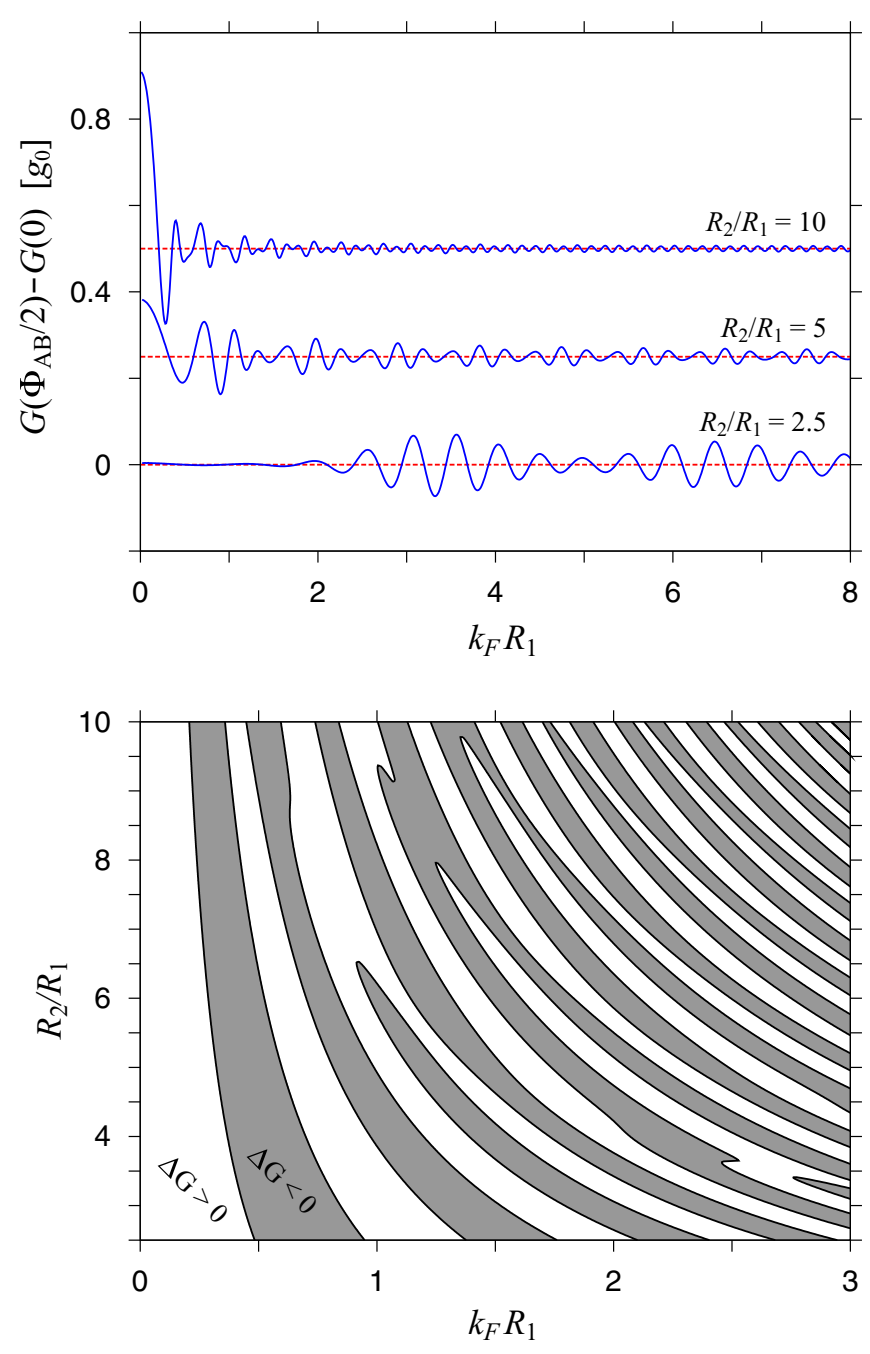

FIG. 3. (Top) Magnitude of the conductance oscillations, $\Delta G=$ $G\left(\Phi_{\mathrm{AB}} / 2\right)-G(0)$, displayed as a function of the doping for selected values of the radii ratio (specified for each line). Notice that the data sets for $R_{2} / R_{1}=5$ and 10 (blue solid lines) are subjected to vertical shifts of 0.25 and $0.5 g_{0}$ (respectively). Red dashed line shows the actual $\Delta G=0$ for each case. (Bottom) Nodal lines (black solid) of $\Delta G$ as a function of the doping and the radii ratio, separating the areas with $\Delta G>0$ (white) and $\Delta G<0$ (shadow).

with a period $\Phi_{\mathrm{AB}}$, at arbitrary doping (see Fig. 2). Quite surprisingly, the magnitude of the conductance oscillations

$$
\Delta G=G\left(\Phi_{\mathrm{AB}} / 2\right)-G(0),
$$

takes relatively large absolute values (namely, $|\Delta G|>0.1 g_{0}$ ) not only in small vicinity of the charge neutrality-point, but also at higher dopings (see Fig. 3), signaling the importance of transport via evanescent waves again. [Notice that the difference between $G\left(\Phi_{\mathrm{AB}} / 2\right)$ and $G(0)$, defining $\Delta G$ via Eq. (16), is governed by only a few modes for which $k_{F} R_{1} \approx \nu(j)$ and thus $T_{j}$ 's are neither $\approx 0$ nor $\approx 1$.] A systematic growth of $\Delta G$ with $R_{2} / R_{1}$ is visible for $k_{F} \rightarrow 0$ (with $\Delta G \approx g_{0}$ for $R_{2} \gg$ $R_{1}$ ), in consistency with earlier predictions of Refs. [2,3] for the uniform magnetic field case.

For each radii ratio, one can find a unique series of discrete doping values for which $\Delta G=0$, resulting in $G\left(\Phi_{i}\right)=$ const. 
For instance, if $R_{2} / R_{2}=5$, the first five nodes of $\Delta G$ correspond to

$$
\left(k_{F} R_{1}\right)_{\Delta G=0}=0.322,0.598,0.814,0.987,1.137 .
$$

Below the first nodal value (i.e., $\left|k_{F} R_{1}\right|<0.322$ ), we have $\Delta G>0$ [or, equivalently, $G\left(\Phi_{\mathrm{AB}}\right)>G(0)$, see Eq. (16)]; then, the sign of $\Delta G$ alternates with growing $k_{F} R_{1}$, as indicated in the bottom panel in Fig. 3.

It is also visible in Fig. 3 that the pattern of nodal lines is rather irregular, as one could expect since $\Delta G$ can be regarded as the rational expression containing Bessel function. Typical separation between the first nodes of $\Delta G$ in Eq. (17) can (roughly) be approximated as $\Delta k_{F} R_{1} \approx 0.3$, which corresponds, for the physical size of $R_{1}=50 \mathrm{~nm}$, to the energy interval of $\Delta E_{F} / k_{B} \approx 40 \mathrm{~K}$ (with the Boltzmann constant $k_{B}$ ). In turn, the conductance oscillations should be observable in comparable or higher temperatures then the standard Aharonov-Bohm effect in graphene rings $[29,30]$.

\section{CONDUCTANCE OSCILLATIONS IN THE ABSENCE OF CYLINDRICAL SYMMETRY}

So far, the discussion was limited to the case of a perfect cylindrical symmetry, allowing us to calculate the transmission probabilities $T_{j}$ [see Eq. (13)] analytically by solving the scattering problem separately for each $(j$ th) angularmomentum mode. In real system, several factors may break the cylindrical symmetry, resulting in the mode mixing. In particular, both the spatial corrugations of a graphene sheet and charge-donating impurities placed in the substrate lead to the charge density fluctuations (i.e., $p$ - $n$ puddles) [16,3133]. For best existing devices, carrier density fluctuations are $\delta n<10^{11} \mathrm{~cm}^{2}$ near the neutrality point, corresponding to the electrostatic potential fluctuation of the order of $\delta U \sim$ $10 \mathrm{meV}$.

Here we test numerically, how robust the effects described in Sec. III are against the cylindrical symmetry breaking. For this purpose, the electrostatic potential energy in the Hamiltonian (4) is replaced by [7]

$$
U(r, \varphi)=-\frac{U_{0} r}{R_{2}} \sin \varphi, \quad R_{1}<r<R_{2} .
$$

In the leads, $r<R_{1}$ or $r>R_{2}$, we set $U(r, \varphi)=U_{\infty}$ again. The potential amplitude (without loss of generality, we suppose $U_{0} \geqslant 0$ ) defines the Fermi energy range, $-U_{0}<E_{F}<$ $U_{0}$, for which a p-n interface is present in the disk area (see Fig. 4). A special case of $U_{0}=0$ restores the uniform-doping case considered in Sec. III.

Regardless of the value of $U_{0}$, angular-momentum eigenfunctions of the form given by Eqs. (6), (11), and (12), still represent the correct solutions in the leads. Therefore the numerical mode matching can be performed in the angularmomentum space, employing the transfer matrix approach presented with details in Ref. [7]. Since the Fermi wave number $k_{F}=\left|E_{F}-U(r, \varphi)\right| /\left(\hbar v_{F}\right)$ is now position-dependent, the numerical results presented in Fig. 4 are parametrized by $E_{F}$ and $U_{0}$. In order to specify these quantities in the physical units, we fixed the disk dimensions at $R_{1}=50 \mathrm{~nm}$ and $R_{2}=5 R_{1}=250 \mathrm{~nm}$ [34]. However, it is worth to stress
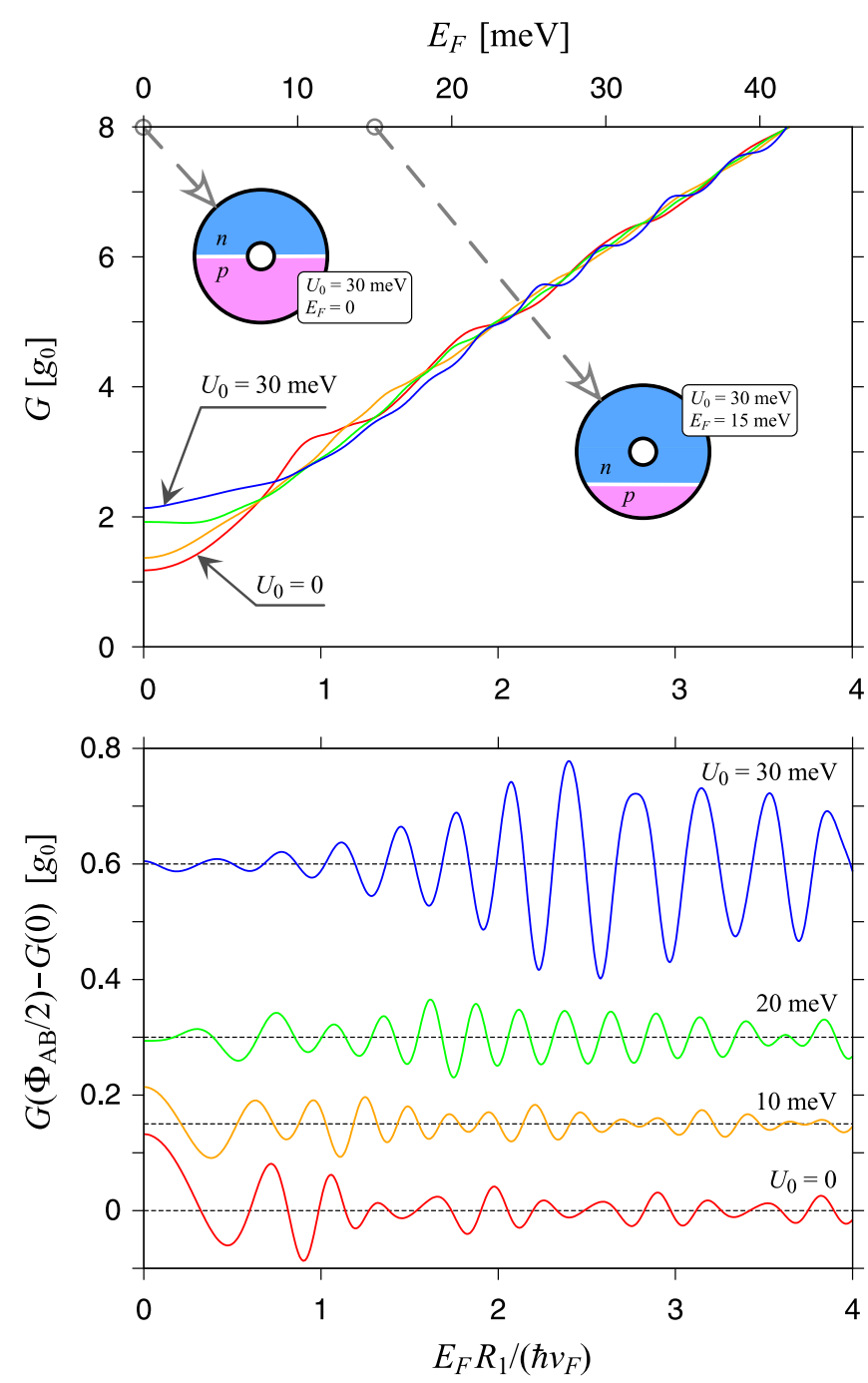

FIG. 4. Conductance for $\Phi_{i}=0$ (top) and the oscillation magnitude (bottom) displayed as functions of the Fermi energy for the disk radii $R_{1}=R_{2} / 5=50 \mathrm{~nm}$ and the electrostatic potential amplitude [see Eq. (18)] varied from $U_{0}=0$ to $30 \mathrm{meV}$ with the steps of $10 \mathrm{meV}$. Top: Two insets show the positions of a $p-n$ interface in the disk area for $U_{0}=20 \mathrm{meV}$ and the two different values of $E_{F}$. (Bottom) The data sets for $U_{0}>0$ (solid lines) are subjected to vertical shifts; black dashed lines show the actual $\Delta G=0$.

that the transport characteristics are determined by the dimensionless parameters, $E_{F} R_{1} /\left(\hbar v_{F}\right)$ (also displayed in Fig. 4), $U_{0} R_{1} /\left(\hbar v_{F}\right)$, and the radii ratio $R_{2} / R_{1}$, and therefore remain invariant upon the scaling $R_{1(2)} \rightarrow \lambda R_{1(2)}, E_{F} \rightarrow E_{F} / \lambda$, and $U_{0} \rightarrow U_{0} / \lambda$, with a real $\lambda>0$.

If the system is close to the charge-neutrality point, namely for $\left|E_{F}\right|<U_{0} R_{1} / R_{2}$, the conductance is noticeably enhanced with growing $U_{0}$ (see top panel in Fig. 4), as the propagation through heavily $p$-doped and $n$-doped areas supplements the transport via evanescent waves. (We further notice that the largest considered $U_{0}=30 \mathrm{meV}$ corresponds to $U_{0} R_{1} /\left(\hbar v_{F}\right) \approx 2.6 \lesssim R_{2} / R_{1}$, and thus the system, at zero field, can be regarded as being in the crossover range between the pseudodiffusive and the ballistic charge transport regimes [35].) For higher $\left|E_{F}\right|$, the effect of $U_{0}$ becomes negligible, 
and $G \approx 2 g_{0}\left\langle k_{F}\right\rangle R_{1}$ with $\left\langle k_{F}\right\rangle=\left|E_{F}\right| /\left(\hbar v_{F}\right)$ being the average Fermi wavenumber on the inner disk edge $\left(r=R_{1}\right)$.

The magnetoconductance oscillations magnitude (see bottom panel in Fig. 4) are diminished for $\left|E_{F}\right|<U_{0} R_{1} / R_{2}$ with growing $U_{0}$. This observation can be rationalized by taking into account that at zero magnetic field main currents flow along the $\varphi \approx \pm \pi / 2$ directions (i.e., towards the regions of extreme doping), for which the magnetic phases associated with the vector potential given by Eq. (5) vanish. In contrast, for the unipolar doping $\left(\left|E_{F}\right|>U_{0}\right)$ the oscillations are only weakly affected by growing $U_{0}$, and the magnitudes of $\Delta G>$ $0.1 g_{0}$ appear for wide range of the doping.

\section{CONCLUSIONS}

We have demonstrated, performing the numerical analysis of the exact formula for transmission probability for electron with a given angular momentum tunneling through the Corbino disk in graphene, that the conductance (as a function of magnetic flux piercing the disk) shows periodic oscillations of the Aharonov-Bohm kind. Unlike for a uniform magnetic field considered in Refs. [2,3], when similar oscillations appear at discrete Landau levels only, the disk in a solenoid magnetic potential shows the oscillations for any
Fermi energy except from a discrete energy set, defined by the disk radii $\left(R_{1}\right.$ and $\left.R_{2}\right)$, the Fermi velocity in graphene $\left(v_{F}\right)$ and the Planck constant $(\hbar)$, for which the conductance is flux-independent.

Most remarkably, away from the charge-neutrality point the conductance oscillations may show a significant magnitude $\left(\Delta G>0.1 g_{0}\right.$, with $\left.g_{0}=4 e^{2} / h\right)$ starting from moderate radii ratios $R_{2} / R_{1} \gtrsim 2$, being comparable to the actual experimental values, see Refs. $[9,10]$. At the charge neutrality point, the oscillation magnitude grows with the radii ratio, approaching $\Delta G \approx g_{0}$ for $R_{2} \gg R_{1}$.

Also, we find out that the conductance oscillations are well-pronounced in the presence of a position-dependent electrostatic potential that breaks the cylindrical symmetry and introduces the mode mixing. Some suppression of the effect is predicted for ambipolar dopings (i.e., with a $p-n$ junction in the disk area), but the oscillations are restored away from the charge neutrality point (for unipolar dopings).

\section{ACKNOWLEDGMENTS}

We thank Piotr Witkowski for discussions. The work was supported by the National Science Centre of Poland (NCN) via Grant No. 2014/14/E/ST3/00256.
[1] A. Rycerz, P. Recher, and M. Wimmer, Phys. Rev. B 80, 125417 (2009).

[2] A. Rycerz, Phys. Rev. B 81, 121404(R) (2010).

[3] M. I. Katsnelson, Europhys. Lett. 89, 17001 (2010).

[4] Z. Khatibi, H. Rostami, and R. Asgari, Phys. Rev. B 88, 195426 (2013).

[5] B. Abdollahipour and E. Moomivand, Physica E 86, 204 (2017).

[6] G. W. Jones, D. A. Bahamon, A. H. Castro Neto, and V. M. Pereira, Nano Lett. 17, 5304 (2017).

[7] D. Suszalski, G. Rut, and A. Rycerz, J. Phys. Mater. 3, 015006 (2020).

[8] J. Yan and M. S. Fuhrer, Nano Lett. 10, 4521 (2010)

[9] E. C. Peters, A. J. M. Giesbers, M. Burghard, and K. Kern, Appl. Phys. Lett. 104, 203109 (2014).

[10] M. Kumar, A. Laitinen, and P. Hakonen, Nat. Commun. 9, 2776 (2018).

[11] Y. Zeng, J. I. A. Li, S. A. Dietrich, O. M. Ghosh, K. Watanabe, T. Taniguchi, J. Hone, and C. R. Dean, Phys. Rev. Lett. 122, 137701 (2019).

[12] C.-I. Liu, D. S. Scaletta, D. K. Patel, M. Kruskopf, A. Levy, H. M. Hill, and A. F. Rigosi, J. Phys. D: Appl. Phys. 53, 275301 (2020).

[13] M. I. Katsnelson, Graphene: Carbon in Two Dimensions (Cambridge University Press, Cambridge, 2012), Chap. 3.

[14] R. Landauer, IBM J. Res. Dev. 1, 223 (1957).

[15] M. Büttiker, Y. Imry, R. Landauer, and S. Pinhas, Phys. Rev. B 31, 6207 (1985).

[16] C. R. Dean, A. F. Young, I. Meric, C. Lee, L. Wang, S. Sorgenfrei, K. Watanabe, T. Taniguchi, P. Kim, K. L. Shepard et al., Nat. Nanotechnol. 5, 722 (2010).
[17] G. Rut and A. Rycerz, Philos. Mag. 95, 599 (2015).

[18] D. A. Siegel, C.-H. Park, C. Hwang, J. Deslippe, A. V. Fedorov, S. G. Louie, and A. Lanzara, Proc. Natl. Acad. Sci. USA 108, 11365 (2011).

[19] C. Hwang, D. A. Siegel, S.-K. Mo, W. Regan, A. Ismach, Y. Zhang, A. Zettl, and A. Lanzara, Sci. Rep. 2, 590 (2012).

[20] L. M. Martelo, M. Dzierzawa, L. Siffert, and D. Baeriswyl, Z. Phys. B 103, 335 (1997).

[21] J. D. Stokes, H. P. Dahal, A. V. Balatsky, and K. S. Bedell, Philos. Mag. Lett. 93, 672 (2013).

[22] D. A. Abanin, I. Skachko, X. Du, E. Y. Andrei, and L. S. Levitov, Phys. Rev. B 81, 115410 (2010).

[23] D. S. Lee, V. Skákalová, R. T. Weitz, K. von Klitzing, and J. H. Smet, Phys. Rev. Lett. 109, 056602 (2012).

[24] More generally, for a centrosymmetric field $\mathbf{B}=B(r) \hat{e}_{z}$, the fraction $\phi=\Phi_{d} / \Phi_{0}$ in Eq. (1) is replaced by

$$
\phi \equiv \frac{\Phi_{i}}{\Phi_{\mathrm{AB}}}+\frac{4 \pi}{\Phi_{0}} \int_{R_{1}}^{R_{2}} \frac{d r}{r} \int_{R_{1}}^{r} d r^{\prime} r^{\prime} B(r),
$$

where $\Phi_{i}$ is flux through the inner ring $\left(r<R_{1}\right)$. In particular, if $B(r)=0$ for $r>R_{1}$, the Aharonov-Bohm-like oscillations occur when varying $\Phi_{i}$.

[25] Yu.V. Nazarov and Ya. M. Blanter, Quantum Transport: Introduction to Nanoscience (Cambridge University Press, Cambridge, UK, 2009), Chap.1.

[26] R. P. Feynman, R. B. Leighton, and M. L. Sands, The Feynman Lectures on Physics (Addison-Wesley, Reading, MA, 1963), Vol. II, Chap. 14. 
[27] We calculated the Hankel functions, $H_{v}(x)^{(1,2)}=J_{v}(x) \pm i Y_{v}(x)$ with $v \geqslant 0$, employing the double-precision regular [irregular] Bessel function of the fractional order $J_{v}(x)\left[Y_{v}(x)\right]$ as implemented in Gnu Scientific Library (GSL), see https://www.gnu. org/software/gsl/doc/html/specfunc.html bessel-functions. For $v<0$, we use $H_{-v}^{(1)}(x)=e^{i \pi v} H_{v}^{(1)}(x)$ or $H_{-v}^{(2)}(x)=e^{-i \pi v} H_{v}^{(2)}(x)$.

[28] G. Nemes, Acta Appl. Math. 150, 141 (2017).

[29] S. Russo, J. B. Oostinga, D. Wehenkel, H. B. Heersche, S. S. Sobhani, L. M. K. Vandersypen, and A. F. Morpurgo, Phys. Rev. B 77, 085413 (2008).

[30] C. Stampfer, E. Schurtenberger, F. Molitor, J. Guettinger, T. Ihn, and K. Ensslin, Int. J. Mod. Phys. 23, 2647 (2009).
[31] Y. Zhang, V. W. Brar, C. Girit, A. Zettl, and M. F. Crommie, Nat. Phys. 5, 722 (2009).

[32] S. Samaddar, I. Yudhistira, S. Adam, H. Courtois, and C. B. Winkelmann, Phys. Rev. Lett. 116, 126804 (2016).

[33] A. Jayaraman, K. Hsieh, B. Ghawri, P. S. Mahapatra, and A. Ghosh, arXiv:2003.02880.

[34] We took the nearest-neighbor hopping integral in graphene $t=2.7 \mathrm{eV}$ and the lattice parameter $a=0.246 \mathrm{~nm}$, leading to $\hbar v_{F}=\sqrt{3} t a / 2=0.575 \mathrm{eV} \mathrm{nm}$.

[35] For $E_{F}=0$, the parameter $u_{0}=U_{0} R_{1}^{2} /\left(R_{2} \hbar v_{F}\right)$ allows one to distinguish between the pseudodiffusive $\left(u_{0} \ll 1\right)$ and the ballistic $\left(u_{0} \gg 1\right)$ charge transport regimes, with crossover occurring for $u_{0} \sim 1$; see also Ref. [7]. 